\title{
Magnetic Coil System for the TCABR Tokamak
}

\author{
E.A. Saettone, A. Vannucci, F.T. Degasperi, R.M.O. Galvão, \\ Y. Kuznetsov, E.K. Sanada, and I.C. Nascimento \\ Laboratório de Física de Plasmas, \\ Instituto de Física, Universidade de São Paulo, \\ São Paulo, CEP 05315-970, SP, Brazil
}

Received on 3 July, 2001

\begin{abstract}
In this work, we discuss the preliminary analysis of some disruptive plasma discharges in the TCABR tokamak, operating in the high density limit. The Fourier analysis of the MHD activity was greatly facilitated because the magnetic coils inside the TCABR were installed as to take into consideration the toroidal geometry of the system, in a straightforward manner. What we have observed is that the $m=3,4$ and 7 MHD components dominate during almost the whole discharge duration, whereas the $m=2$ MHD mode increased substantially just before the occurrence of a major disruption. Also, we could estimate the angular velocity of the magnetic islands, which was observed to increase up to three times just before the major disruption.
\end{abstract}

\section{Introduction}

It has been experimentally proved that the use of magnetic coils are essential for determining the MHD components, responsible for triggering the disruptive phenomenon in tokamaks. The disruption instability imposes severe restrictions on the plasma current and particle density [1], causing a sudden expansion of the plasma column, which is followed by a large negative spike in the $V_{\text {loop }}$ signal, in spite of the apparent normal evolution of the plasma discharge [2]. It is usually preceded by intense MHD activity (Mirnov oscillations).

Theoretically, the basis on which this instability occurs in tokamaks is not totally well known, yet. Usually, the high density disruptions are understood as being triggered by an interaction between magnetic islands [3]. Experimentally, in most tokamaks it has been observed that the $m=2$ MHD component (corresponding to the magnetic islands located within the surface $q=2$ ) grow exponentially, triggering the disruption.

\section{Experimental set-up}

The TCABR tokamak is a middle size tokamak with the main parameters showed in Table 1 . The magnetic coil system is composed of 22 magnetic probes, installed around one rectangular cross section inside the vacuum vessel of the tokamak [4], as shown in Fig. 1. Each probe has two windings, perpendicular to each other, one in the $R$ direction and the other one in the $Z$ di- rection.

\begin{tabular}{lcc} 
TABLE 1. Main parameters of the TCABR tokamak. \\
\hline Toroidal Magnetic Field & $B_{T}$ & $1.07 \mathrm{~T}$ \\
Major Radius & $R_{o}$ & $0.61 \mathrm{~m}$ \\
Minor Radius & $a$ & $0.18 \mathrm{~m}$ \\
Plasma Current & $I_{p}$ & $100 \mathrm{kA}$ \\
Discharge Duration & $\tau_{d}$ & $150 \mathrm{~ms}$ \\
Electronic Central Density & $n_{e}$ & $3 \times 10^{19} \mathrm{~m}^{-3}$ \\
Electronic Central Temperature & $T_{e}$ & $400 \mathrm{eV}$ \\
\hline
\end{tabular}

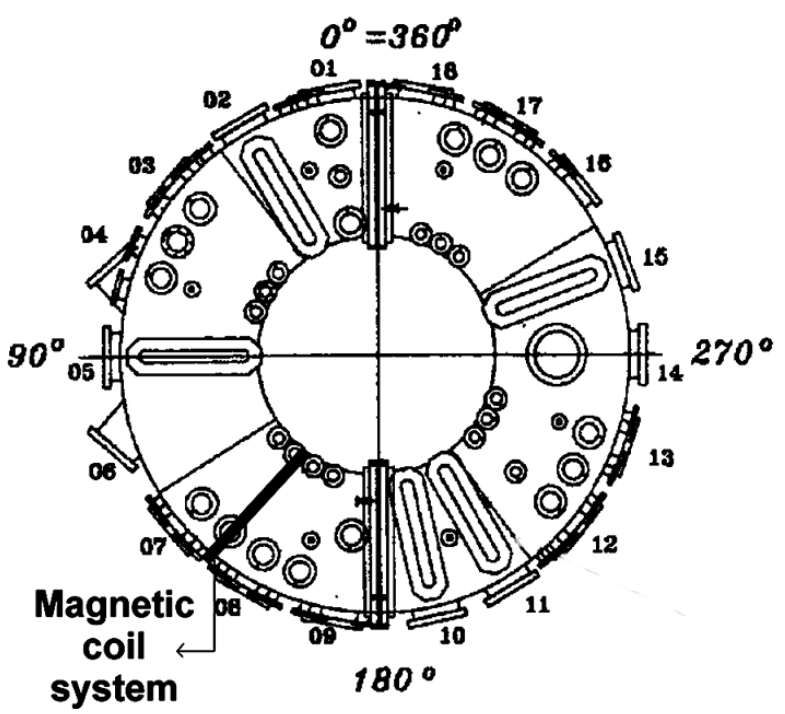

Figure 1. Upper view of the TCABR tokamak, showing the rectangular cross section where the magnetic coil system is installed. 
The positioning of the magnetic probes was chosen as to take into account automatically the toroidal effects of the system due the toroidal geometry of the tokamak (Fig. 2). This correction was introduced by following the angular distribution given by the Merezhkin correction, [5]

$$
\theta^{*}=\theta+\lambda \sin \theta
$$

where $\theta$ is the poloidal angle considering (in the cylindrical approximation) an equal angular distribution for the coils, and $\lambda$ is given by the following expression [5]:

$$
\lambda=\frac{r_{m n}}{R_{m n}}\left[\beta_{p}\left(r_{m n}\right)+\frac{1}{2} \ell_{i}\left(r_{m n}\right)+1\right]
$$

where $\beta_{p}$ is the beta poloidal and $l_{i}$ is the internal inductance of the confined plasma.

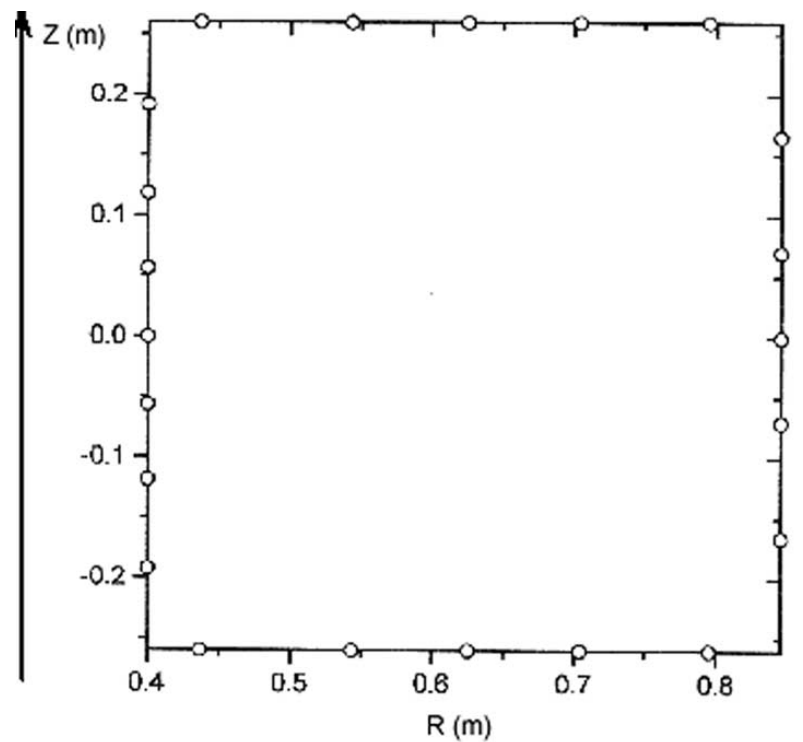

Figure 2. Rectangular cross section of the tokamak showing the position of each magnetic probe, distributed in the poloidal direction, taking into account the toroidal effect due to the toroidal geometry.

The effective area (sensibility) of each coil was experimentally determined by creating an oscillating magnetic field using a Helmholtz coil, with amplitude of $1.8 \times 10^{-5} T$. The frequency range of the oscillating magnetic field was chosen between $5 \mathrm{kHz}$ and $40 \mathrm{kHz}$.

During the experiments, all the 44 probe signals were bandpass filtered, between $1 \mathrm{kHz}$ and $100 \mathrm{kHz}$, and amplified electronically. Afterwards, these signals were digitalized and saved using the TCAqs (acquisition data system of the TCABR tokamak), with $250 \mathrm{kHz}$ of acquisition sampling rate. A computer program was constructed to Fourier analyse the experimental data and to calculate the dominant MHD modes present on the disruptive TCABR discharges analyzed.

\section{Experimental data treatment}

Some procedures have been followed to treat the experimental data from the magnetic coil system, using a computer program. First, the program considers the effective area of the coils, obtained from the calibration and, afterwards, it calculates the magnetic field composition measured by the two coils of each probe, in the poloidal direction.

Also, the computer program calculates the angular position of every probe (equation (1)), using $\lambda$ from the equation (2), which is measured from the vertical equilibrium field $\left(B_{v}\right)$, as given by the equation [6]:

$$
B_{v}=\frac{\mu_{o} I_{p}}{4 \pi r_{p}}\left[\ln \left(\frac{8 r_{p}}{a_{p}}\right)+\Lambda-\frac{1}{2}\right]
$$

where $\Lambda$ is the Shafranov parameter, defined by

$$
\Lambda=\beta_{p}+\frac{\ell_{i}}{2}-1
$$

where $\beta_{p}$ is the beta parameter and $l_{i}$ is the internal inductance of the plasma. Therefore, for the TCABR tokamak, equation (2) can be written as

$$
\lambda=\frac{a}{R}\left(\beta_{p}^{T C A B R}+\frac{3}{2}\right)
$$

where the $\beta_{p}^{T C A B R}$ corresponds to the beta parameter as measured from the MHD equilibrium. The computer program also considers that the perturbed magnetic field, as measured by the Mirnov coils, has a dependence with the distance given by:

$$
\dot{\tilde{B}}_{m}\left(\theta_{k}\right) \propto \frac{1}{r_{k}^{m+1}}
$$

where $\dot{\tilde{B}}_{m}\left(\theta_{k}\right)$ is the experimental data, as measured by the probe at the $\theta_{k}$ poloidal angle, and $r_{k}$ is the ratio $a / b_{k}$, being $a$ the plasma column radius and $b_{k}$ the distance between the plasma column center and the magnetic coil located at angular position $\theta_{k}$ (which is different for each probe because of the rectangular cross section of the conducting walls). To calculate the contribution of each MHD mode, in each plasma discharges, the program uses the following expression [7]:

$$
\dot{\tilde{B}}_{m}\left(\theta_{k}\right)=A_{m} \cos \left(m \theta_{k}\right)+B_{m} \sin \left(m \theta_{k}\right)
$$

where the Fourier constants $A_{m}$ and $B_{m}$ are calculated by the following equations [7]:

$$
\begin{aligned}
& A_{m}=\frac{1}{11}\left[\sum_{k=1}^{22} \dot{\tilde{B}}_{m}\left(\theta_{k}\right) \cos \left(m \theta_{k}\right)\right] \\
& B_{m}=\frac{1}{11}\left[\sum_{k=1}^{22} \dot{\tilde{B}}_{m}\left(\theta_{k}\right) \sin \left(m \theta_{k}\right)\right]
\end{aligned}
$$

Finally, the computer program creates a new set of $c o r$ rected poloidal signals, which are then Fourier analyzed in a straightforward manner. 


\section{Analysis of a disruptive dis- charge}

In the TCABR tokamak, disruptive discharges are obtained whenever the machine is operated at the density limit $\left(\bar{n}_{e} \geq 3 \times 10^{19} \mathrm{~m}^{-3}\right)$. During these plasma discharges, plasma currents of approximately $80 \mathrm{kA}$ are obtained for about $50 \mathrm{~ms}$, until the occurrence of a major disruption. Typically, the loop voltage is about $2 V$, with no significant $H_{\alpha}$ emission or hard $X-$ ray emission. The experimental magnetic signals picked up by the Mirnov coils, however, show typical Mirnov oscillations, with frequencies in the range of $10 \mathrm{kHz}$ to $15 \mathrm{kHz}$.

Fig. 3 shows the experimental signals of a discharge (shot No 2017) in the high density limit. It is observed a major disruption in $t=86.8 \mathrm{~ms}$, that annihilate the plasma in $\tau \sim 20 \mathrm{~ms}$. Note that the Mirnov oscillations have a constant amplitude during almost the whole discharge, until the disruption takes place (between $t \sim 50 \mathrm{~ms}$ and $t \sim 85 \mathrm{~ms}$ ), which indicates a saturation of the magnetic islands. The safety factor for this discharge was calculated to be $q(a) \approx 4.4$.

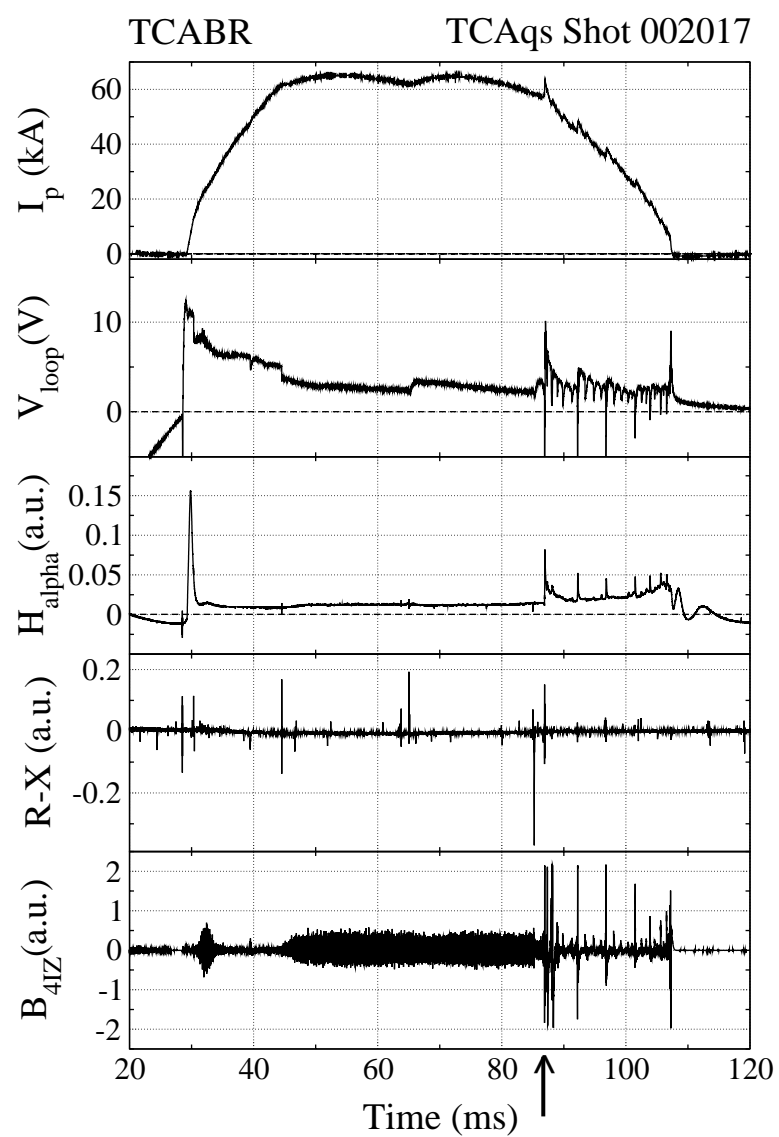

Figure 3. Shot No 2017 of the TCABR tokamak operating in the high density limit. A major disruption occurs in $\mathrm{t}=86.8 \mathrm{~ms}$ (arrow at the bottom). After that, the plasma current decays to zero in about $20 \mathrm{~ms}$.

The same experimental signals, now expanded around the disruption time, are shown in Fig. 4. Observe that at $t=85.2 \mathrm{~ms}$, the amplitude of the MHD activity decreases considerably, at the same time a spike is observed in the hard X-ray non-integrated signal. After that, in $t=86.3 \mathrm{~ms}$, the Mirnov oscillations amplitude begins to grow exponentially, during approximately $0.5 \mathrm{~ms}$, until the occurrence of the disruption in $t=86.8 \mathrm{~ms}$.

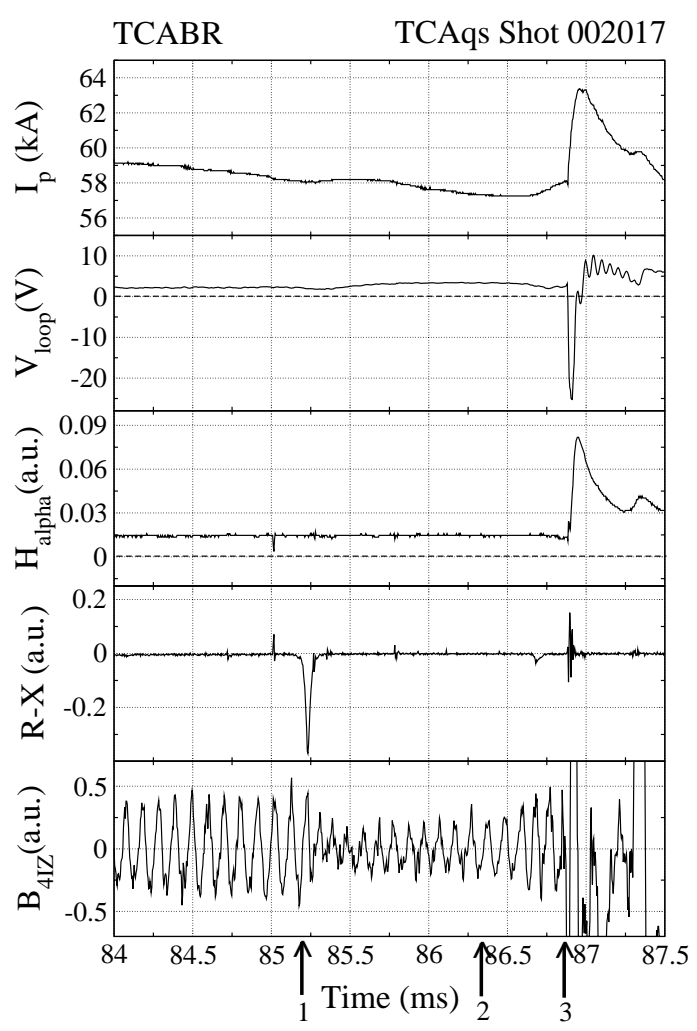

Figure 4. Shot No 2017 of the TCABR tokamak expanded in time around the occurrence of the disruption. In $t=85.2$ ms (arrow No 1), MHD activity decreases considerably. In $\mathrm{t}=86.3 \mathrm{~ms}$ (arrow No 2), the Mirnov oscillation amplitude grows exponentially until disruption occurs, in $\mathrm{t}=86.8 \mathrm{~ms}$ (arrow No 3).

Experimentally, the exponential growth rate of the MHD activity, just before the disruption, was calculated to be $\gamma_{\exp }=8.9 \times 10^{3} \mathrm{~s}^{-1}$. Comparing this value to the theoretical resistive growth rate, calculated to be $\gamma_{\text {res }}=3.1 \times 10^{4} s^{-1}$, (the theoretical value of the ideal growth rate was calculated to be $\gamma_{\text {ideal }}=1.3 \times 10^{6} s^{-1}$ ) the disruptive event in this discharge could be explained in terms of an interaction between magnetic islands. The frequency of the precursor oscillations was also observed to increase from $10 \mathrm{kHz}$ to $13 \mathrm{kHz}$, in approximately $0.2 \mathrm{~ms}$ before the disruption takes place. This probably means that the angular velocity of magnetic islands also increased substantially during this short period of time. 
As to compare the evolution of the MHD poloidal modes existing in this plasma discharge, we Fourier analyzed the experimental signals between $t=75 \mathrm{~ms}$ and $t=76 \mathrm{~ms}$, when there was no evidence yet of any precursor to the disruptive instability. It was then observed that the MHD modes $m=3,4$ and 7 were dominant (Fig. 5), and the angular velocity of the magnetic islands, between these two instants of time, was estimated to be $\omega_{\text {rot }}=1.6 \times 10^{3} \mathrm{rad} / \mathrm{s}$.
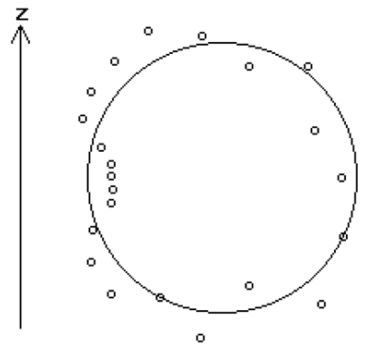

(a)

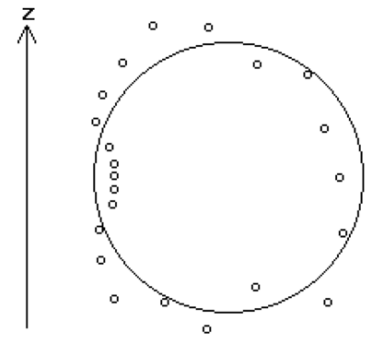

(b)

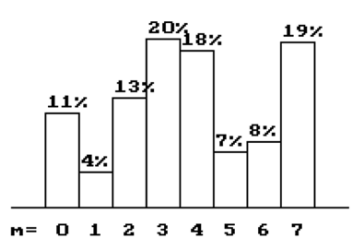

Figure 5. Polar diagrams (left) and MHD spectra (right), calculated at (a) $\mathrm{t}=75 \mathrm{~ms}$ and $(\mathrm{b}) \mathrm{t}=76 \mathrm{~ms}$, for the shot No 2017 of the TCABR tokamak.

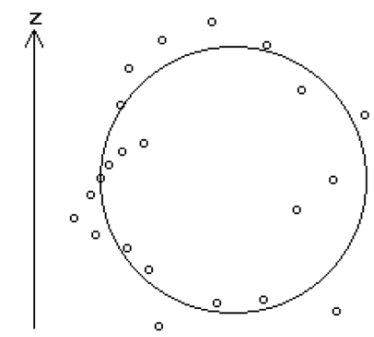

(a)
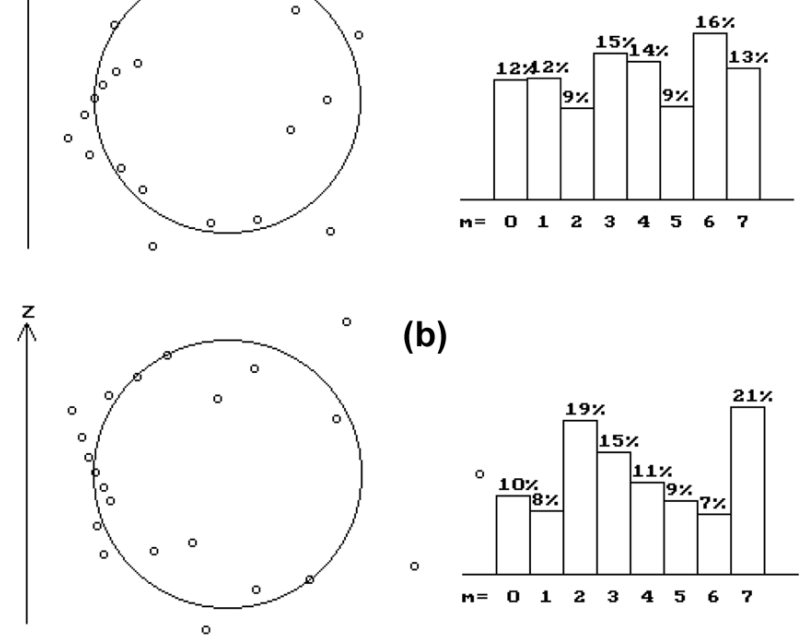

(b)

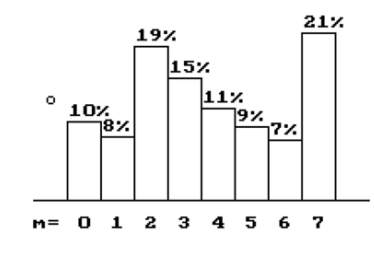

Figure 6. Polar diagrams (left) and MHD spectra (right), calculated at (a) $\mathrm{t}=86.3 \mathrm{~ms}$ and $(\mathrm{b}) \mathrm{t}=86.75 \mathrm{~ms}$, of the shot No 2017 of the TCABR tokamak.

The same calculations were also carried out just before disruption, for the same plasma discharge. As observed in Fig. 6-b, the MHD modes corresponding to $m=2$ and $m=7$ are clearly dominant, just before the disruption $(t=86.75 \mathrm{~ms})$. The angular velocity of the magnetic islands just before disruption, was estimated to be $\omega_{\text {rot }}=5.0 \times 10^{3} \mathrm{rad} / \mathrm{s}$. New coils are being positioned inside the vaccum vessel, in different toroidal position, to verify the $n$ number for these modes.

\section{Conclusions}

Some disruptive discharges, that occurred when the TCABR is operated at the density limit, were analized in this article. It was observed that the Mirnov oscillations, along the discharge, have a frequency of $10 \mathrm{kHz}$. It was also determined that the $m=3,4$ and 7 MHD modes dominates during the whole discharge, with an angular velocity of the corresponding magnetic islands estimated to be $1.6 \times 10^{3} \mathrm{rad} / \mathrm{s}$.

However, just before disruption (approximately $0.2 \mathrm{~ms}$ before the occurrence of the disruption), it was observed that the Mirnov oscillations frequency increases up to $13 \mathrm{kHz}$. At this time, a larger contribution of the $m=2$ MHD component was calculated, which probably was the responsible for triggering the disruption. The angular velocity for the corresponding magnetic islands was estimated to be $5.0 \times 10^{3} \mathrm{rad} / \mathrm{s}$, three times more than during the rest of the discharge.

Finally, the exponential growth rate of the Mirnov oscillations was compared to the theoretical values, related to the resistive and ideal growth rates, from which we inferred that the occurrence of the disruption could be explained in terms of a magnetic islands interaction.

\section{Acknowledgments}

This work has been partially supported by FAPESP and by the Ministry of Science and Technology through the PRONEX program. Also we want to thanks Mr. Nelson M. Cuevas, Mr. Ablício Pires dos Reis and Juan I. Elizondo for the technical support.

\section{References}

[1] S.V. Mirnov, I.B. Semenov, Soviet Physics JETP, 33, 11347 (1971).

[2] J.A. Wesson, MHD Stability Theory, Academic Press, 1981, pp.191-233.

[3] G. Bateman, MHD Instabilities, MIT Press, New York, 1978, pp. 265-278.

[4] I. Semenov, S. Mirnov, et al., Review of Scientific Instruments, 70, 449 (1999).

[5] T.R. Harley, D.A. Buchenauer, J.W. Coonrod, K.M. McGuire, Nucl. Fus., 29, 771 (1989).

[6] Yu.K. Kuznetsov, I.N. El Chamaa, I.C. Nascimento, R.M.O. Galvão, Physics of Plasmas, 6, 4002 (October 1999).

[7] M.S.T. Araújo, "MHD, Programa para a Obtenção da Evolução Temporal das Componentes MHD do Plasma do Tokamak TBR-1", Report IFUSP/P-1000 (1992). 